\title{
Branching defects
}

DOI:

10.1038/nrc2134

\section{URLs \\ Kras \\ http://www.ncbi.nlm.nih.gov/ entrez/query.fcgi?db=gene\&c $\mathrm{md}=$ RetrieveEdopt=full_ reportElist_uids=16653 \\ Spry2 \\ http://www.ncbi.nlm.nih.gov/ entrez/query.fcgi?db=gene\&c $\mathrm{md}=$ Retrieve\&dopt=full reportElist_uids $=24064$}

The Ras genes are some of the most frequently mutated genes in human cancer, but despite decades of study many aspects of Ras function - and dysfunction - have still to be fully elucidated. This is probably due to the complexity of the Ras signalling network, which is modulated in a context-dependent manner by various cross-talk and feedback mechanisms that have not yet been studied in detail. Now Alice Shaw,

Tyler Jacks and colleagues show that a Ras-mitogen-activated protein kinase (MAPK) antagonist, Sprouty 2 (Spry2), regulates oncogenic Kras during development and tumorigenesis.

As Ras-activating mutations underlie both neoplastic and developmental disorders, the authors studied the effects of an oncogenic Kras $^{\mathrm{G} 12 \mathrm{D}}$ mutation during mouse development and found that germline $\operatorname{Kras}^{\mathrm{G} 12 \mathrm{D}}$ activation resulted in early embryonic lethality owing to a defective placental trophoblast. Although this could be rescued by restricting $\mathrm{Kras}^{\mathrm{G} 12 \mathrm{D}}$ expression to the embryo proper, the embryos still died of cardiovascular and haematopoietic defects, and showed a severe lung branching defect, having persistent dilated bronchi with few terminal bronchi or bronchioles. Lung branching morphogenesis is regulated by signals from both the lung epithelium and the underlying mesenchyme. The authors found that mice that express Kras ${ }^{\mathrm{G} 12 \mathrm{D}}$ only in the developing lung epithelium had abnormal lungs, indicating that $\mathrm{Kras}^{\mathrm{G} 12 \mathrm{D}}$ affects the epithelial rather than the mesen- chymal compartment.

To gain an insight into the molecular mechanisms that cause defective branching, the authors looked at Spry2 - a key regulator of branching. In situ hybridization analyses showed that the expression of Spry 2 was increased and more uniformly distributed in $\mathrm{Kras}^{\mathrm{G} 12 \mathrm{D}}$ fetal lungs, compared with wild-type lungs in which high Spry2 expression levels were restricted to the leading edge of lung branching tips. As Spry2 is an antagonist of Ras-MAPK signalling, Jacks and colleagues looked at MAPK activation using anti-phoshoMAPK antibodies. The total level of phoshoMAPK did not change significantly, but its localization was altered, mirroring that of Spry2, suggesting that oncogenic Ras impairs lung branching through the Spry2-mediated disruption of phosphoMAPK localization. Indeed, both the in vivo knockout of Spry2 and its knockdown, through lentiviral short hairpin RNA, suppressed the lung branching defect induced by oncogenic Kras.

Given that the expression of oncogenic Kras also induces lung cancer, might Spry2 also be involved? The expression of SPRY2 was increased in lung tumours from mice that expressed a $\mathrm{Kras}^{\mathrm{G} 12 \mathrm{D}}$ allele that is sporadically activated by spontaneous recombination, and SPRY2 levels inversely correlated with those of phosphoMAPK. Moreover, Spry2-null mice showed an increased number of lung tumours induced by Kras $^{\mathrm{G} 12 \mathrm{D}}$ expression, underpinning the idea that
Spry2 functions as a tumour suppressor in $\mathrm{Kras}^{\mathrm{G} 12 \mathrm{D}}$-mediated lung tumorigenesis.

Although the mechanisms by which Spry2 antagonizes Ras signalling are complex and context dependent, this work indicates the importance of negative regulation in the pathogenesis of Ras-based disease.

Francesca Pentimalli

ORIGINAL RESEARCH PAPER Shaw, A. T. et al. Sprouty-2 regulates oncogenic K-ras in lung development and tumorigenesis. Genes Dev. 21, 694-707 (2007)

FURTHER READING Schubbert, S., Shannon, K. \& Bollag, G. Hyperactive Ras in developmental disorders and cancer. Nature Rev. Cancer 7 , 295-308 (2007)

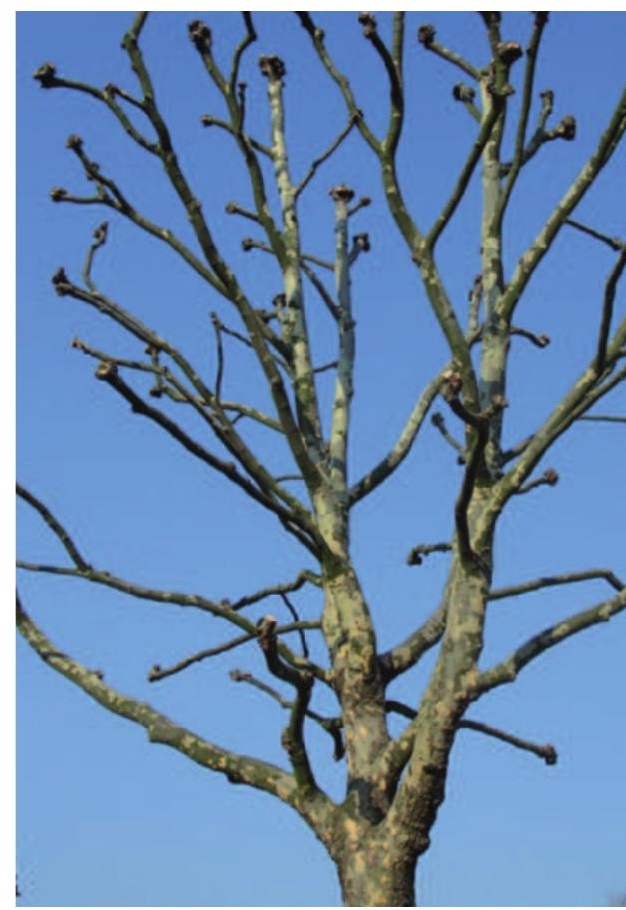

